\title{
1 Dynamics of silver elution from functionalised antimicrobial
}

\section{2 nanofiltration membrane}

3 S. Choudhari, ${ }^{\text {ab }}$ O. Habimana, ${ }^{\text {c }}$ J. Hannon,,${ }^{\mathrm{d}}$ A. Allen, ${ }^{\mathrm{a}}$ E. Cummins ${ }^{\mathrm{d}}$ and

4 E. Casey ${ }^{\text {a* }}$

5 a. School of Chemical and Bioprocess Engineering, University College Dublin (UCD),

6 Belfield, Dublin 4, Ireland.

$7 \quad$ b. Department of Biological Sciences, Dayananda Sagar University, Kumarswamy

8 Layout, Bangalore 560078, India.

9 c. School of Biological Sciences, The University of Hong Kong, Hong Kong, PR China.

10 d.School of Biosystems and Food Engineering, Agricultural and Food Science Centre,

11 University College Dublin (UCD), Belfield, Dublin 4, Ireland.

$12 *$ Corresponding author. Mailing address: University College Dublin, School of

13 Chemical and Bioprocess Engineering, Belfield, Dublin 4, IRELAND. Phone: +3531

14716 1877, Email: eoin.casey@ucd.ie

$15+$ Both authors contributed equally to this work.

18 Word Count:

19 Text: 5510

20 References: 933

21 Figures: 260

22 Tables: 69 


\section{Dynamics of silver elution from functionalised antimicrobial nanofiltration membrane}

In an effort to mitigate biofouling on thin film composite membranes such as nanofiltration and reverse osmosis, a myriad of different surface modification strategies has been published. The use of silver nanoparticles (Ag-NPs) has emerged as being particularly promising. Nevertheless, the stability of these surface modifications is still poorly understood, particularly under permeate flux conditions. Leaching or elution of Ag-NPs from the membrane surface can not only affect the antimicrobial characteristics of the membrane, but it could also potentially present an environmental liability when applied in industrial-scale systems. This study sought to investigate the dynamics of silver elution and the bactericidal effect of an Ag-NP functionalised NF270 membrane. Inductively Coupled Plasma-Atomic Emission Spectroscopy was used to show that the bulk of leached silver occurred at the start of experimental runs, and was found to be independent of salt or permeate conditions used. Cumulative amounts of leached silver did, however, stabilise following the initial release, and were shown to have maintained the biocidal characteristics of the modified membrane, as observed by a higher fraction of structurally damaged Pseudomonas fluorescens cells. These results highlight the need to comprehensively assess the timedependent nature of bactericidal membranes.

Keywords: Nanofiltration; Silver modification, leaching, ICP, biofouling

\section{Introduction}

Biofouling remains a major operating problem in reverse osmosis and nanofiltration processes (Flemming et al. 1997, Gutman et al. 2012, Ridgway et al. 1983, Vrouwenvelder and Van der Kooij 2001). Biofilms are at the core of the problem and their recalcitrance leads to performance loss, the use of significant quantities of cleaning chemicals and/or accelerated membrane deterioration. An increasing number of publications propose various forms of membrane functionalisation in an effort to manage the biofouling problem and these are comprehensively reviewed (Adeleye et al. 2016, Kang and Cao 2012, Rana and Matsuura 2010). 
functionalised membranes, their translation to full-scale operation has not occurred. A number of possible reasons include: diminished membrane flux and/or salt retention, the use of toxic or expensive chemicals, complex membrane manufacturing processes, and poor long-term stability under full-scale process conditions.

Amongst the most promising anti-biofouling technologies is the use of biocidal

60 metal nanoparticles (Ben-Sasson et al. 2016, Dolina et al. 2015, Dror-Ehre et al. 2010,

61 Liu et al. 2013, Mauter et al. 2011, Perkas et al. 2013, Suresh et al. 2013, Zhu et al. 2016, Zodrow et al. 2009) with a particular interesting approach proposed by Ben-

63 Sasson and collegues (Ben-Sasson et al. 2014) where the biocidal activity of the

64 membrane can be regenerated in situ. Regardless of the methodology used for the

65 modification of membrane surfaces with compounds such as silver, the stability of these surface modifications is poorly understood. Measurement of low concentrations of metal nanoparticles in membrane module effluent is a difficult process, requiring expensive analytical methods such as Inductively Coupled Plasma Spectroscopy (ICP). Moreover, when this type of characterisation is performed, it requires a thorough 70 preparation procedure involving acid digestion, which renders the sample unusable for further characterisation with alternative techniques.

To the best of the author's knowledge, there are no studies that have examined

73 the time-dependent nature of silver leaching from silver modified membranes in ideal

74 and realistic effluent under cross-flow conditions in combination with bactericidal

75 assessment. The present study aimed to address this knowledge gap using an established membrane functionalisation technique (Ben-Sasson et al. 2014). 


\section{Model Synthetic Water.}

79 Grade 1 quality water $\left(18.2 \mathrm{M} \Omega \mathrm{cm}^{-1}\right.$, pH $\left.6.01 \pm 0.11\right)$ obtained from an Elga

80 Process Water System (Biopure 15 and Pureflex 2, Veolia, Ireland), was used

81 throughout this study and will henceforth be referred to as Milli-Q water (Semiao

82 et al. 2013). A synthetic water solution was prepared by dissolving the following

83 salts in Milli-Q water: sodium bicarbonate $\left(\mathrm{NaHCO}_{3}\right) 0.0042 \mathrm{~g} \mathrm{~L}^{-1}$, sodium

84 chloride $(\mathrm{NaCl}) 0.0117 \mathrm{~g} \mathrm{~L}^{-1}$, potassium phosphate $\left(\mathrm{KH}_{2} \mathrm{PO}_{4}\right) 0.0063 \mathrm{~g} \mathrm{~L}^{-1}$,

85 magnesium sulphate (sold as heptahydrate, $\mathrm{MgSO}_{4} \cdot 7 \mathrm{H}_{2} \mathrm{O}$ ) $0.015 \mathrm{~g} \mathrm{~L}^{-1}$,

86 ammonium chloride $\left(\mathrm{NH}_{4} \mathrm{Cl}\right) 0.005 \mathrm{~g} \mathrm{~L}^{-1}$, and calcium chloride (sold as

87 dihydrate, $\left.\mathrm{CaCl}_{2} \cdot 2 \mathrm{H}_{2} \mathrm{O}\right) 0.0076 \mathrm{~g} \mathrm{~L}^{-1}$. All salts were purchased in their pure form,

88 or in the annotated hydrate form, from Sigma-Aldrich, Ireland. This synthetic

89 water will henceforth be referred to as Raw water. Prior to experiments, model

90 Raw water $10 \mathrm{~L}$ batches were prepared.

\section{Filtration membrane.}

93 The nanofiltration membrane samples used in this study were cut from a single

94 large flat-sheet of NF270 membrane (Dow Filmtec, USA). The NF270 membrane

95 is a thin film composite polyamide nanofiltration membrane used in the drinking

96 water industry. After 18 hours of filtering Milli-Q water at 15 bar, the compacted

97 membrane samples yielded a steady Raw water flux rate of $118.5 \pm 8.5 \mathrm{~L} \mathrm{~m}^{-2} \mathrm{hr}^{-1}$

98 with a $94.8 \pm 0.8 \%$ retention of salts at 15 bar and $20^{\circ} \mathrm{C}$. Rectangular membrane

99 samples, $27 \mathrm{~cm} \times 5 \mathrm{~cm}$, were cut from the flat-sheet roll and soaked overnight in 
100 Milli-Q water at $4{ }^{\circ} \mathrm{C}$ to remove their preservative layer. The membranes were

101 finally rinsed thoroughly with Milli-Q water.

102 Silver modifications of NF270 membranes. The membranes were modified following

103 step-by-step procedures detailed in recent studies (Ben-Sasson et al. 2014). The method

104 chosen for this study can be considered as a very promising technique in that it allows

105 for the regeneration of the biocidal activity of membranes in-situ. The detailed

106 mechanisms for immobilisation are further described in a series of articles (Joly et al.

107 2000, Li et al. 2006, Tiraferri and Elimelech 2012). In brief, NF270 membrane sheets

108 (Dow Filmtec, USA) were cut into smaller sized sheets and fitted into a custom-made

109 Nylon $10 \mathrm{~cm}$ x $20 \mathrm{~cm}$ frame, designed for membrane surface modification reactions.

110 The membranes were first soaked at $4{ }^{\circ} \mathrm{C}$ in deionised water overnight for the removal

111 of membrane preservatives. Soaked membranes were individually placed in a nylon

112 frame with the membrane active surface layer exposed for ensuing modifications.

113 Membrane modification and preparation consisted of the following steps: (i) a 10-

114 minute exposure with silver nitrate $\left(\mathrm{AgNO}_{3}\right) 5 \mathrm{mM}$ solution by pouring $50 \mathrm{~mL}$ into the

115 frame unit and allowing the solution to react with the active layer of the membrane in

116 the dark by covering the frame with aluminium foil. The membrane surface known for

117 its negatively charged carboxylic groups (Tiraferri and Elimelech 2012), interacts with

118 silver ions, thereby establishing electrostatic pairs on the membranes surface. The

119 presence of such electrostatic pairs is necessary for subsequent nucleation of silver

120 nanoparticles (Ag-NPs) at a later stage of the modification process (Joly et al. 2000, Li

121 et al. 2006) ; (ii) the careful removal of the $\mathrm{AgNO}_{3}$ solution from the nylon frame and

122 membrane surface, which left a thin layer on the membrane surface; (iii) a 5 minute

123 reaction with $\mathrm{NaBH}_{4}$, by pouring a $50 \mathrm{~mL} 5 \mathrm{mM}$ solution onto the freshly silver

124 conditioned membrane, for the immobilization of (Ag-NPs) on the membrane's active 
125 layer. The addition of $\mathrm{NaBH}_{4}$ solution leads to the immediate reduction of free silver

126 ions to Ag-NPs on the membrane surface according to (Song et al. 2009); (iv) the

127 careful removal of $\mathrm{NaBH}_{4}$ solution and the removal of unreacted $\mathrm{NaBH}_{4}$ residues from

128 the surface of the modified active layer, by soaking the modified membranes two

129 separate times in $50 \mathrm{~mL}$ deionised (DI) water; and (v) the removal of silver modified

130 membrane from its nylon frame and rinsing in deionised (DI) water for $30 \mathrm{~s}$ to remove

131 any loosely bound Ag-NPs from the membrane's surface, prior to storage in the dark at

$1324{ }^{\circ} \mathrm{C}$ before use.

134 Surface quantification and characterisation of silver modified membranes.

135 X-ray photoelectron spectroscopy (XPS) analysis was performed to assess the

136 presence of silver using an electron spectrometer (Kratos Analytical, Manchester,

137 United Kingdom) equipped with a monochromated Al Ka-ray source. XPS survey

138 spectra were collected in the binding energy range of 0-1200 eV. Photoelectrons

139 were detected at $90^{\circ}$ take- off angle (TOA) and the corresponding depth of

140 analysis was $10 \mathrm{~nm}$. The accuracy of XPS was determined to be at $\pm 2 \%$. The

141 centre of tested dehydrated silver-modified and pristine NF270 membrane

142 samples was used for the XPS analysis and each result presented in the present

143 work is an average of three measurements taken at different locations. Membrane

144 performance of silver-modified NF270 membranes was assessed through pure

145 water flux and permeates conductivity measurements using Milli-Q or Raw water.

146 Following an initial 2-hour compaction period at 15 bar using Milli-Q water, pure

147 water flux measurements were obtained at 3 bar pressure conditions at a cross

148 flow velocity of $0.66 \mathrm{~L} \mathrm{~min}^{-1}$. Salt retention measurements were obtained by 
149 measuring the conductivity of permeate and Raw water feed following 3 bar

150 pressure conditions at a cross flow velocity of $0.66 \mathrm{~L} \mathrm{~min}^{-1}$ at $20 \pm 0.5^{\circ} \mathrm{C}$.

152 Silver leaching experiments.

153 Silver leaching experiments on NF270 modified membranes were performed in

154 cross flow systems and Membrane Fouling Simulators (MFS) (Vrouwenvelder et

155 al. 2006) under both non-flux and flux conditions at constant $\mathrm{pH}$ and temperature

156 conditions.

157 Silver leaching under non-permeate flux conditions was assessed using either

158 Milli-Q or Raw water medium at a cross velocity of $0.037 \mathrm{~m} \mathrm{~s}^{-1}, \operatorname{Re}=14.32$ and a

159 shear rate of $53.6 \mathrm{~s}^{-1}$ in each MFS cell in continuous mode (Supplementary figure

160 1). At least $5 \mathrm{~mL}$ of Milli-Q and Raw water samples were sampled emanating

161 directly from individual MFS cells and were collected at 0-, 0.5-, 2-, 4- and 21-

162 hour time points for silver quantification analyses. Likewise, at least $5 \mathrm{~mL}$ of

163 accumulated Milli-Q or Raw water samples in waste containers were also

164 collected following 0.5-, 1-, 2-, 4- and 21-hour experimental runs in continuous

165 mode. The sample containers were covered with aluminium foil to avoid exposing

166 accumulated water volumes to light. Prior to collection, containers were shaken.

167 Experiments were repeated in duplicate with independently silver-modified

168 NF270 membranes.

169 Under nanofiltration conditions, the degree of leaching on silver modified NF270

170 membranes was assessed following membrane compaction and salt conditioning

171 steps commonly used prior to standard bio-adhesion and biofouling experiments

172 (Semiao et al. 2013). The experimental setup was designed as previously

173 described (Heffernan et al. 2014). Briefly, the cross-flow system was composed of 
174 a 10 L autoclavable Nalgene feed tank (VWR Ireland) and a high-pressure pump

175 (Hydra-Cell, UK). The system was connected to three MFS devices placed in

176 parallel, two of which contained single independently silver-modified NF270

177 membranes and one MFS with a pristine NF270 control membrane. Temperature

178 was monitored in the feed tank with a temperature indicator (Pt 100, Radionics,

179 Ireland) and maintained at $20^{\circ} \mathrm{C} \pm 1^{\circ} \mathrm{C}$ with a coil inside the tank connected to a

180 temperature controlled MultiTemp III water bath (Pharmacia Biotech, Ireland). A

181 back-pressure regulator (KPB1L0A415P20000, Swagelok, UK) allowed the

182 pressurisation of the system up to the required pressure. The pressure was

183 monitored in both feed and retentate side of the membrane cells with two pressure

184 transducers (PTX 7500, Druck, Radionics, Ireland). The feed flow was measured

185 using a flow meter (OG2, Nixon Flowmeters, UK). Data-logging was set-up

186 allowing for data collection of membrane cells inlet and outlet pressure, feed flow

187 rate and temperature (PicoLog 1000, PicoTechnology, Radionics, Ireland).

188 Permeate flux and permeate conductivity measurements were performed

189 throughout the compaction experiment and salt conditioning steps of the

190 experiment. To assess the level of remnant silver on membranes during

191 nanofiltration, compaction was first conducted at 15 bar using Milli-Q water for 2

192 hours at a cross-flow velocity of $0.66 \mathrm{~L} \mathrm{~min}^{-1}$ per MFS. Following the two-hour

193 compaction period, the system was temporarily stopped to allow the removal of a

194 MFS device containing one silver-modified membrane from the rig, and the

195 replacement of the Milli-Q water tank with a tank containing 10 L Raw water.

196 The salt conditioning step was then initiated for the two remaining MFS devices

197 at 3 bar (conditions used for the bioassay experiment) for $30 \mathrm{~min}$ at a cross flow

198 velocity of $0.66 \mathrm{~L} \mathrm{~min}^{-1}$. Following salt conditioning, the system was stopped and 
a second MFS device containing a silver-modified NF270 removed from the system. Membranes were removed from their MFS devices and membrane samples were cut into $1 \mathrm{~cm}^{2}$ area, in preparation for silver quantification analysis. This experiment was repeated in duplicate using independent silver-modified membranes. Moreover, two independent un-filtered silver-modified membranes were used as controls to establish the level of silver on modified membranes prior to nanofiltration.

Silver quantification through Inductively Coupled Plasma-Atomic Emission Spectroscopy (ICP-AES).

209 The concentration of total silver (ionic silver and Ag-NPs) contained in Milli-Q

210 water and Raw water following contact with membranes under shear and static

211 conditions was determined by ICP-AES using a recently described standard

212 operating procedure (Hannon et al. 2016). A blank and four calibration standards

$213\left(1,5,10\right.$ and $\left.20 \mu \mathrm{g} \mathrm{L}^{-1}\right)$ were created by serial dilution from a $\mathrm{AgNO}_{3}$ standard

214 (1000 mg L ${ }^{-1} \mathrm{Ag}+$ in $\mathrm{HNO}_{3}$, Elementec, Ireland). To detect potential matrix

215 effects, yttrium (1000 mg L ${ }^{-1} \mathrm{Y}$ in $\mathrm{HNO}_{3}$, Elementec, Ireland) was spiked in all

216 standards and samples. To ensure the absence of cross contamination between

217 samples, all glassware was soaked for 24 hours in $5 \% \mathrm{HNO}_{3}$ in distilled water,

218 followed by rinsing with a copious amount of distilled water and drying in a

219 closed glassware dryer. Reagent blanks run between samples confirmed the 220 absence of silver contamination in the system. To test the accuracy and precision 221 of the method over the entire calibration range, fortified reagent blanks $(2,10$ and 
$22220 \mu \mathrm{g} \mathrm{L}^{-1}$ in Raw water) were prepared in a similar manner to calibration

223 standards, and recoveries were found to be $88 \%, 107 \%$ and $89 \%$, respectively.

224 To determine the amount of silver leaching from modified NF270 membranes, 5

$225 \mathrm{~mL}$ water samples were prepared for ICP-AES analysis by acidifying with $100 \mu \mathrm{L}$

226 of $69 \% \mathrm{HNO}_{3}$ (VWR International, Ireland) and $50 \mu \mathrm{L}$ of $37 \% \mathrm{HCl}$ (Sigma-

227 Aldrich, Ireland). For the total digestion of autopsy membrane samples, each

228 membrane was sliced into $1 \mathrm{~mm} \times 1 \mathrm{~mm}$ pieces and placed in a PTFE vessel with

$22910 \mathrm{~mL} 69 \% \mathrm{HNO}_{3}$. Digestion was carried out for 5 hours at $120{ }^{\circ} \mathrm{C}$. Following

230 digestion, $100 \mu \mathrm{L}$ of membrane digestate was diluted in $9.9 \mathrm{~mL}$ of Milli-Q water

231 to ensure the silver concentration fell within the calibration range before ICP-AES

232 analysis.

233

234 Cell preparation for adhesion assay.

235 A Gram-negative fluorescent mCherry-expressing Pseudomonas fluorescens

236 PCL1701 (Lagendijk et al. 2010) were selected as the model strain in this study.

237 Pseudomonas cultures were stored at $-80{ }^{\circ} \mathrm{C}$ in King B broth (King et al. 1954)

238 supplemented with $20 \%$ glycerol. Cultured $P$. fluorescens were obtained by

239 inoculating $100 \mathrm{~mL}$ concentrated Raw water $\left(\mathrm{Na}_{3} \mathrm{C}_{6} \mathrm{H}_{5} \mathrm{O}_{7} \cdot 2 \mathrm{H}_{2} \mathrm{O} 1.3 \mathrm{~g} \mathrm{~L}^{-1}\right.$,

$240 \mathrm{NaHCO}_{3} 0.042 \mathrm{~g} \mathrm{~L}^{-1}, \mathrm{NaCl} 0.117 \mathrm{~g} \mathrm{~L}^{-1}, \mathrm{KH}_{2} \mathrm{PO}_{4} 0.063 \mathrm{~g} \mathrm{~L}^{-1}, \mathrm{MgSO}_{4} \cdot 7 \mathrm{H}_{2} \mathrm{O} 0.15$

$241 \mathrm{~g} \mathrm{~L}^{-1}, \mathrm{NH}_{4} \mathrm{Cl} 0.05 \mathrm{~g} \mathrm{~L}^{-1}, \mathrm{CaCl}_{2} \cdot 2 \mathrm{H}_{2} \mathrm{O} 0.076 \mathrm{~g} \mathrm{~L}^{-1}$ ) supplemented with gentamicin

242 (Sigma-Aldrich, Ireland) at a final concentration of $10 \mu \mathrm{g} \mathrm{mL}^{-1} \mathrm{using}$ single

243 colonies previously grown on King B agar (Sigma-Aldrich, Ireland) at $28^{\circ} \mathrm{C}$. 
244 Subsequently, cultures were incubated overnight at $30{ }^{\circ} \mathrm{C}$ with shaking at 200

$245 \mathrm{rpm}$ and left to grow overnight.

246 A Staphylococcus epidermidis (ATCC 12228) was also selected as a Gram-

247 positive model strain. Staphylococcus cultures were stored at $-80{ }^{\circ} \mathrm{C}$ in King B

248 broth (King et al. 1954) supplemented with $20 \%$ glycerol. Cultured $S$.

249 epidermidis were obtained by inoculating $100 \mathrm{~mL}$ King B broth, using single

250 colonies previously grown on King B agar (Sigma-Aldrich, Ireland) at $28{ }^{\circ} \mathrm{C}$.

251

252 Static bioadhesion assays.

253 Cell concentrations were standardised for each strain and adhesion experiment by

254 first centrifuging $P$. fluorescens and S. epidermidis overnight cultures at $5000 \mathrm{rpm}$

255 for 10 min using a Hettich Universal 320R centrifuge (Lennox, Ireland), and by

256 re-suspending cell pellets in sterile PBS. Cell suspensions were then diluted to an

$257 \mathrm{OD}_{600}$ of 0.2 in $40 \mathrm{~mL}$, which corresponds to an inoculum of approximately $10^{8}$

258 cells $\mathrm{mL}^{-1}$.

259 Static adhesion assays were performed on both silver modified and pristine

260 NF270 membranes. Membrane samples were cut into small sections and

261 immobilised at the bottom of 6-well plates (Nunc, Roskilde, Denmark). Bacterial

262 adhesion was initiated by adding $4 \mathrm{~mL}$ of freshly prepared cell suspensions of $P$.

263 fluorescens or S. epidermidis cells in individual wells. Wells were then statically

264 left to rest for $30 \mathrm{~min}$ at room temperature. To end the adhesion experiments, 4

$265 \mathrm{~mL}$ sterile PBS solution was added to individual wells, followed by a systematic

266 removal of a $4 \mathrm{~mL}$ volume of diluted bacterial suspension. This process was 
repeated three times for each well of the 6-well plate. Static adhesion was

268 performed three times using independent $P$. fluorescens or $S$. epidermidis cultures.

270 Viability analysis and epi-florescence Microscopy analysis.

271 To assess the degree of cell structural damage on adhered cells following

272 bioadhesion assays, a volume of $1 \mu \mathrm{L}$ of SYTOX Green $(5 \mathrm{mM})$ (Invitrogen,

273 Dublin, Ireland) was added to individual wells of the 6-well plates containing $P$.

274 fluorescens cells. Damaged S. epidermidis cells were stained by adding $1 \mu \mathrm{L}$

275 Propidium Iodide (PI) (20 mM) (Invitrogen, Dublin, Ireland). For visualising total

276 adhered $S$. epidermidis cells, $1 \mu \mathrm{L}$ DNA-based Syto 9 stain $(5 \mathrm{mM})$ (Invitrogen,

277 Dublin, Ireland) was introduced to relevant wells. Stained wells were

278 subsequently incubated at ambient temperature for $10 \mathrm{~min}$ in the dark prior to epi-

279 fluorescence microscopy (Olympus BX51) using a 10X objective. Two images

280 were acquired for every chosen observation field using U-MNG and U-MWB

281 filter cubes for differentiating between fluorescent mCherry-tagged and SYTOX

282 Green-stained Pseudomonas cells. In the case of Staphylococcus cells, the U-

283 MNG and U-MWB filter cubes were utilised to visualise PI-positive and Syto-9

284 positive cells, respectively. Ten different fields of view were obtained at random

285 points from each tested membrane sample. Cell surface coverage (\%) for

286 mCherry-tagged, SYTOX Green-, PI-, and Syto 9- stained cells was determined

287 for each tested membrane using ImageJ® software, a Java-based image

288 processing program (http://rsbweb.nih.gov/ij/). 
291 To assess the degree of structural damage incurred by the presence of silver on the modified NF270 membrane, compared to adhesion onto pristine membranes

293 following static adhesion or adhesion under permeate flux conditions, cells were

294 prepared for scanning electron microscopy. Following epi-fluorescence

295 microscopy, fouled membrane samples were chemically fixated and dehydrated in

296 their respective wells. Submerged membrane samples were fixed by adding

297 glutaraldehyde to a final concentration of $2.5 \%$ and left to incubate overnight. All

298 samples were then rinsed with $0.1 \mathrm{M} \mathrm{NaCl}$ solution then gradually dehydrated

299 using increasing volumes of ethanol for 5min intervals until samples were

300 submerged in $100 \%$ ethanol. The wells were then emptied and the samples were

301 left to dry.

302

303 Statistical analysis.

304 The statistical significance of the presence of silver on NF270 surfaces on the

305 viability of $P$. fluorescens or $S$. epidermidis following 30 min bioassays conducted

306 in static conditions was assessed using One-way analysis of variance with

307 MINITAB v15.1 (Minitab Inc., State College, PA, USA). All tests for

308 significance were performed assuming a null hypothesis of equality with a

309 difference deemed significant at $\mathrm{P}<0.05$. Statistics are presented as "test type

310 (degrees of freedom $)=$ test statistic, $\mathrm{P}<$ value". 


\section{Results and Discussion}

\section{Surface characterisation of modified membrane.}

314 XPS analysis was performed on silver modified NF270 membranes to confirm the

315 presence of silver following the membrane surface modification protocol chosen for this

316 study. XPS is a useful analytical tool for determining the atomic composition of specific

317 functional groups or elements in the first $10 \mathrm{~nm}$ of any dehydrated surface. The presence

318 of silver on the modified membrane surface was confirmed by the presence of a

319 characteristic peak at $368 \mathrm{eV}$ (Figure 1) (Stobie et al. 2008) and the evaluated atomic \%

320 of silver on the membrane surface were found to be $1.43 \%$. Unmodified, pristine

321 NF270 membranes exhibited peaks at 531, 399 and $284 \mathrm{eV}$, which correspond to O $1 \mathrm{~s}$,

322 N 1 s and C 1 s respectively. Similarly, to virgin NF270 membranes, silver modified

323 membrane exhibited all the above-mentioned peaks, as these are the main constituent

324 elements of the polyamide layer of NF270 membranes. As expected, the peak for silver

325 at $368 \mathrm{eV}$ was absent in pristine NF270 membranes. These results are indicative of the

326 successful silver modification of NF270 membranes and validate the surface

327 modification protocol used in this study.

328 One of the major consequences of membrane surface modifications can be linked to the

329 potential reduction of membrane permeability and salt retention properties. It was

330 therefore necessary to evaluate pure water permeability and salt rejection of silver

331 modified membranes. The pure water flux and salt rejection $\%$ of pristine and modified

332 membrane are presented in table 1 . The pure water flux results were shown to have not

333 been significantly altered by the presence of Ag-NPs on the membrane surface

334 compared to pristine NF270 membranes with flux rate values of values of $33.1 \mathrm{~L} \mathrm{~m}^{-2} \mathrm{hr}$

$335{ }^{-1}$ and $34.4 \mathrm{~L} \mathrm{~m}^{-2} \mathrm{hr}^{-1}$, respectively. Likewise, membrane salt rejection showed to have

336 not been affected by the presence of Ag-NPs on the surface of NF270 membranes 
compared to pristine control membranes, with salt rejection values of $57.8 \%$ and 59.3

$338 \%$, respectively. Despite the insignificant decrease in membrane performance, changes

339 attributed to the presence of Ag-NPs can be considered negligible. Observed variations

340 in this study could have been ascribed by the intrinsic disparities between the

341 individually tested membranes. This result also shows that the surface modifications did

342 not affect the intrinsic properties of the selective polyamide layer of NF270 membranes.

\section{Bioadhesion Experiment}

345 The antimicrobial properties of silver modified NF270 was first tested against Gram-

346 negative (Pseudomonas fluorescens) and Gram-positive (Staphylococcus epidermidis)

347 by performing controlled bioadhesion assays under static conditions (Figure 2). $P$.

348 fluorescens cells were found to have larger surface coverage on pristine NF270

349 membranes $(\mathrm{P}<0.0001)$ and silver modified membranes $(\mathrm{P}<0.0001)$ compared to $S$.

350 epidermidis cells (Figure 2a).

351 These differences can be attributed to the intrinsic differences between the $P$.

352 fluorescens and S. epidermidis cells in terms of their cell surface hydrophobicity or cell

353 surface electronegativity (Allen et al. 2015, Kiers et al. 2001, Vanloosdrecht et al.

354 1987). When comparing the surface coverage variations between tested membranes,

355 mCherry-expressing $P$. fluorescens cells was found to be lower $(\mathrm{P}<0.0001)$ on silver-

356 modified NF270 membranes compared to pristine membranes with surface coverage

357 values at $13.9 \%$ and $26.4 \%$ respectively. No difference in surface coverage $(\mathrm{P}=0.99)$

358 was observed for Syto-9 stained S. epidermidis cells adhered on pristine and silver-

359 modified NF270 membranes with values of $7.0 \%$ and $6.8 \%$ respectively.

360 These results suggest that Gram-negative cells are more sensitive to the tested

361 silver modified NF270 membrane surface compared to Gram-positive cells. Although 
362 silver has long been recognised as a potent antimicrobial, the mechanism behind its

363 bactericidal activity is still not clearly elucidated. Interestingly, a recent study showed

364 that silver is able to not only break down key bacterial cellular metabolic processes, but

365 can also contribute to cell wall damage by initiating the production of reactive oxygen

366 species. This causes membrane permeability particularly in Gram-negative bacteria

367 compared to Gram-positive bacteria, making the former more susceptible to a broad

368 range of secondary treatment in the form of antibiotics (Morones-Ramirez et al. 2013).

369 This was substantiated by examining the physiological state of the adhered cells

370 following the bioadhesion static assay by using a dye that positively labelled cells with

371 compromised cell wall membranes (Figure 2b). Results demonstrated that Gram-

372 negative cells were more susceptible $(\mathrm{P}=0.0016)$ on silver-modified NF270 compared to

373 Gram-positive cells, with dead-to-live cell ratios of 0.14 and 0.068 , respectively. When

374 comparing these ratios to pristine NF270 membranes, the silver modified NF270

375 membranes produced a significantly higher ratio of dead cells in Gram-negative cells

$376(\mathrm{P}<0.0001)$, while no effect was observed for Gram-positive cells $(\mathrm{P}=0.5279)$.

377 Considering that the silver-modified NF270 was most effective against Gram-

378 negative cells, it was found to be essential to test these membranes under nanofiltration

379 process conditions, and to establish whether the antimicrobial mechanism of these

380 membranes was caused by the sole presence of antimicrobial compounds on the

381 membrane's surface or a potential combination of the environments present under

382 nanofiltration.

383 One recent study showed that the high shear and permeate flux conditions

384 experienced at the membrane surface was significant enough to cause cell wall

385 structural damage and cell collapse of adhering P. fluorescens cells (Habimana et al.

386 2014). The data in table 2 show that no differences in surface coverage were observed 
387 for $P$. fluorescens cells following 3 bar nanofiltration on pristine and silver modified

388 membranes $(\mathrm{P}=0.2471)$, with surface coverage values of $8.3 \%$ and $8.7 \%$ respectively.

389 However, silver modified membranes led to higher ratios $(\mathrm{P}<0.0001)$ of dead to live $P$.

390 fluorescens cells following nanofiltration on silver modified membranes compared to

391 pristine NF270 membranes with values of approximately 0.71 compared to 0.43 ,

392 respectively. This higher presence of dead cells on silver modified NF270 signifies its

393 potential application in impeding biofouling in accordance with previously published

394 characterisation using the same type of functionalisation (Ben-Sasson et al. 2014) .

395 Future studies will assess whether the initial higher presence of dead cells on the surface

396 of silver modified membranes will impede or accelerate the rate of biofouling compared

397 to fouling on pristine membranes.

$398 \quad$ These studies will be essential for determining whether silver based

399 modifications of nanofiltration membranes have a prospect of being applied as a

400 strategy for controlling and managing biofouling.

$401 \quad$ The higher ratio of dead P. fluorescens cells on silver-modified NF270

402 membrane following bioassays under nanofiltration processes was corroborated by

403 qualitative analysis using high-resolution electron microscopy (Figure 3), revealing

404 noticeable cell-wall structural damage. Compared to pristine NF270 membranes (Figure

405 3AB), P. fluorescens cells appeared to have completely collapsed on silver modified

406 membranes (Figure 3CB). Damaged cells were also observed on pristine NF270

407 following nanofiltration (Figure 3B), as previously described in a recent study

408 (Habimana et al. 2014), where bacterial cells adhering to membranes under

409 nanofiltration conditions are stressed by permeate drag forces, affecting their structural

410 integrity. 
However, the presence of totally collapsed $P$. fluorescens cells on silver-

412 modified surfaces suggests that the silver at the membrane surface albeit at low

413 concentrations (based on XPS results) can alter the structural integrity of the adhered

414 cells. Coupled with the high concentration of salts at the membrane interface caused by

415 concentration polarisation, the silver antimicrobial effect could have been amplified

416 during nanofiltration processes. One recent study on antibacterial properties of silver

417 ions showed that in the presence of carbonate ions, the antimicrobial activity of silver

418 ions was enhanced by a factor of up to 1,000 (Swathy et al. 2014). This synergistic

419 effect was suggested to act in two steps which involve a first removal of peripheral

420 bacterial cell wall proteins targeted by salt ions, facilitating the binding of silver onto

421 the bacterial outer membrane (Swathy et al. 2014). With the weakened cell wall

422 structure under extreme hydrodynamic conditions during nanofiltration process, the

423 bacterial cell may have collapsed on silver-modified NF270 membranes.

\section{Silver stability/leaching experiment.}

425 The silver stability and its release profile of were crucial elements in this study, since

426 leaching or elution of Ag-NPs from surface-modified membranes can negatively impact

427 the originally intended level of antimicrobial efficacy, while also presenting potential

428 environmental liability with the levels of released Ag-NPs. Moroever, the loss of silver

429 from modified surfaces could also lead to incurred compounded costs needed for silver

430 regeneration procedures of membranes. Most of the previous studies documented do not

431 provide insights into the stability of modified membranes under permeate flux

432 conditions. The majority of studies demonstrate silver leaching effects either under

433 static conditions or with the use of DI water, which for the most part, are not fully

434 representative of actual operating conditions. One recent study involving covalently

435 attached Ag-NP thin-film composite (TFC) membranes, tested the stability of silver 
436 modified surfaces was tested in in DI water (Yin et al. 2013). In another study, silver

437 leaching effects on Ultra Filtration (UF) membranes modified with different forms of

438 silver modifictaion were studied using with DI water at 1 bar and crossflow velocity of

$4393 \mathrm{~L} \mathrm{~min}^{-1}$ (Dolina et al. 2015). In a more recent study, silver leaching under static and

440 filtration conditions was also investigated using DI water (Liu et al. 2016). It goes

441 without saying that testing the stability of surface-modified NF membranes should be

442 performed under representative nanofiltration conditions. In the case of silver-modified

443 NF membranes, to the best of the author's knowledge, there are no substantial studies in

444 which the degree of silver removal due to permeate flux conditions is assessed. In this

445 study, both the effects of environmental salinity and nanofiltration process conditions

446 on the leaching of silver from membranes was determined in controlled sets of

447 experiments.

448 A substantial portion of the total leached silver occurred almost immediately

449 following the start of the flow. The lack of variation in the amount of released silver

450 between Milli-Q and Raw water environments within the first hour of the experimental

451 run suggests that the effect of salinity of the environment to which membranes were

452 exposed was insignificant in the observed leaching phenomenon. The amount of silver

453 leached following the initial first hour of the experiment stabilised over the course of

454 21-hour runs. Over longer periods, exposure to Milli-Q water led to higher amounts of

455 leached silver compared to membranes exposed to Raw-water, which caused a small

456 reduction in the amount of released silver (results not shown). This difference although

457 insignificant could have been attributed to the variation in the original silver loading

458 between tested membranes, considering that the membranes were independently

459 modified. 
461 quantity of released silver was observed within the first 4 hours of exposure to Milli-Q

462 or Raw water. The cumulative amount of released silver however stabilised between 4

463 and 21 hours, suggesting that the leaching effect had stabilised in that period. These

464 results affirm the need to not only assess the leaching phenomena in relevant

465 experimental conditions, but also, help determine the dynamics of leaching. The latter is

466 most relevant in experiments involving complex environments such as in nanofiltration

467 processes where shear and concentration polarization effects may impact on the rate of 468 leaching.

469 This study demonstrates an insignificant difference of silver leaching from silver

470 modified membranes under ideal (Milli-Q) and realistic (Raw water) environmental

471 conditions. Although the greatest quantity of silver leaching has been found to occur

472 within a short period of time and further transient leaching is not anticipated beyond a

473 24-hour period, long term release studies over the membranes useful life time in

474 combination with bactericidal assessment should be performed to elucidate potential

475 leaching phenomenon.

476 With growing interest in the potential application of functionalised nanofiltration

477 membranes as an antifouling strategy, special focus should be placed in the

478 characterisation of the stability of chemically modified surfaces within relevant

479 experimental contexts. This is particularly important when testing novel antifouling

480 molecules with antifouling or antimicrobial properties on membranes. In this study, the

481 effects of membrane compaction and salt conditioning of membranes under permeate

482 flux conditions on silver leaching was assessed (Figure 5), as these experimental steps

483 are established prerequisites for fundamental bioadhesion studies during nanofiltration.

484 Surprisingly, surface silver concentration was found to be no different to that of non- 
compacted (control) modified membrane following compaction with Milli-Q.

486 Considering that the compaction procedure was performed in recirculation, the leached

487 silver may have been re-adsorbed onto the membrane surface over the course of the experiment. Likewise, following compaction and Raw water salt conditioning, changes in membrane surface silver concentration was found to be insignificant. The observed

490 lack of variability of membrane silver-content during nanofiltration suggests that the

491 recirculation environment within the system may have led to the re-deposition of silver onto the membrane during experiments. This hypothesis should be tested in subsequent studies in which collected permeate is prohibited from recirculating within the system and the cumulative silver content quantified and compared with the silver content in the 495 feed.

This study would be necessary to assess the environmental risk of chemically modified nanofiltration membranes through the likely trans-membrane transport of leached antimicrobials into the permeate. Moreover, the recirculation of leached silver may potentially create uncertainties on the antimicrobial dynamics that occur at the

500 membrane surface. It is for this reason that an emphasis needs to be placed on the

501 testing of antimicrobial properties of surface modified membranes under realistic conditions.

\section{Conclusion}

505 The bactericidal effect of silver nanoparticle modification of NF270 has been

506 demonstrated and is in alignment with findings from a previously publication using the

507 same membrane functionalisation method. However, the purpose of this study was to

508 explore the dynamics of the system under permeate flux conditions. Based on 
510 the ionic environment performed or pressure conditions used in this study. Moreover,

511 significant silver leaching occurred at the start of experimental runs at the onset of

512 shear, but stabilised over time. Despite a relatively high initial release of silver,

513 modified membranes still preserved their biocidal properties.

514

\section{Acknowledgements}

516 This research was supported by the European Research Council (ERC), project 278530,

517 funded under the EU Framework Programme 7. The authors would like to thank Mr. Pat

518 O'Halloran for his invaluable technical assistance, and Mr. Liam Morris for the

519 construction of the MFS devices. The authors especially thank Dr. Ellen L. Lagendijk

520 from the Institute of Biology Leiden, Netherlands for the gift of the Pseudomonas

521 fluorescens PCL1701. Dr. Ian Reid from UCD's Nano Imaging and Material Analysis

522 Centre (NIMAC) is acknowledged for his assistance with XPS analysis.

\section{References}

524 Adeleye AS, Conway JR, Garner K, Huang Y, Su Y, Keller AA. 2016. Engineered

525 nanomaterials for water treatment and remediation: Costs, benefits, and applicability.

526 Chemical Engineering Journal.286:640-662.

527 Allen A, Semiao AJC, Habimana O, Heffernan R, Safari A, Casey E. 2015.

528 Nanofiltration and reverse osmosis surface topographical heterogeneities: Do they

529 matter for initial bacterial adhesion? Journal of Membrane Science. Jul 15;486:10-20.

530 Ben-Sasson M, Lu X, Nejati S, Jaramillo H, Elimelech M. 2016. In situ surface

531 functionalization of reverse osmosis membranes with biocidal copper nanoparticles.

532 Desalination.388:1-8.

533 Ben-Sasson M, Lu XL, Bar-Zeev E, Zodrow KR, Nejati S, Qi GG, Giannelis EP, 534 Elimelech M. 2014. In situ formation of silver nanoparticles on thin-film composite 535 reverse osmosis membranes for biofouling mitigation. Water Research. Oct 1;62:260536270. 
537 Dolina J, Dlask O, Lederer T, Dvorak L. 2015. Mitigation of membrane biofouling 538 through surface modification with different forms of nanosilver. Chemical Engineering 539 Journal. Sep 1;275:125-133.

540 Dror-Ehre A, Adin A, Markovich G, Mamane H. 2010. Control of biofilm formation in 541 water using molecularly capped silver nanoparticles. Water Research.44:2601-2609.

542 Flemming H-C, Schaule G, Griebe T, Schmitt J, Tamachkiarowa A. 1997. Biofouling543 the Achilles heel of membrane processes. Desalination.113:215-225.

544 Gutman J, Fox S, Gilron J. 2012. Interactions between biofilms and NF/RO flux and 545 their implications for control-A review of recent developments. Journal of Membrane 546 Science.421:1-7.

547 Habimana O, Semiao AJC, Casey E. 2014. Upon Impact: The Fate of Adhering 548 Pseudomonas fluorescens Cells during Nanofiltration. Environ Sci Technol. Aug 549 19;48:9641-9650.

550 Hannon JC, Kerry JP, Cruz-Romero M, Azlin-Hasim S, Morris M, Cummins E. 2016. 551 Assessment of the migration potential of nanosilver from nanoparticle-coated low552 density polyethylene food packaging into food simulants. Food Addit Contam A. Jan 553 2;33:167-178.

554 Heffernan R, Habimana O, Semiao AJC, Cao H, Safari A, Casey E. 2014. A physical 555 impact of organic fouling layers on bacterial adhesion during nanofiltration. Water 556 Research. Dec 15;67:118-128.

557 Joly S, Kane R, Radzilowski L, Wang T, Wu A, Cohen R, Thomas E, Rubner M. 2000. 558 Multilayer nanoreactors for metallic and semiconducting particles. Langmuir.16:13545591359.

560 Kang G-d, Cao Y-m. 2012. Development of antifouling reverse osmosis membranes for 561 water treatment: a review. Water Research.46:584-600.

562 Kiers PJM, Bos R, van der Mei HC, Busscher HJ. 2001. The electrophoretic softness of 563 the surface of Staphylococcus epidermidis cells grown in a liquid medium and on a 564 solid agar. Microbiol-Uk. Mar;147:757-762.

565 King EO, Ward MK, Raney DE. 1954. Two simple media for the demonstration of 566 pyocyanin and fluorescin. Journal of laboratory and clinical medicine.44:301-307. 
567 Lagendijk EL, Validov S, Lamers GE, De Weert S, Bloemberg GV. 2010. Genetic tools

568 for tagging Gram-negative bacteria with mCherry for visualization in vitro and in

569 natural habitats, biofilm and pathogenicity studies. FEMS microbiology letters.305:81-

57090.

571 Li Z, Lee D, Sheng X, Cohen RE, Rubner MF. 2006. Two-level antibacterial coating

572 with both release-killing and contact-killing capabilities. Langmuir.22:9820-9823.

573 Liu SS, Zhang MY, Fang F, Cui L, Wu JJ, Field R, Zhang KS. 2016. Biogenic silver

574 nanocomposite TFC nanofiltration membrane with antifouling properties. Desalin

575 Water Treat. May 14;57:10560-10571.

576 Liu Y, Rosenfield E, Hu M, Mi B. 2013. Direct observation of bacterial deposition on 577 and detachment from nanocomposite membranes embedded with silver nanoparticles.

578 Water Research.47:2949-2958.

579 Mauter MS, Wang Y, Okemgbo KC, Osuji CO, Giannelis EP, Elimelech M. 2011.

580 Antifouling ultrafiltration membranes via post-fabrication grafting of biocidal

581 nanomaterials. ACS applied materials \& interfaces.3:2861-2868.

582 Morones-Ramirez JR, Winkler JA, Spina CS, Collins JJ. 2013. Silver Enhances

583 Antibiotic Activity Against Gram-Negative Bacteria. Sci Transl Med. Jun 19;5.

584 Perkas N, Lipovsky A, Amirian G, Nitzan Y, Gedanken A. 2013. Biocidal properties of

585 TiO2 powder modified with Ag nanoparticles. J Mater Chem B.1:5309-5316.

586 Rana D, Matsuura T. 2010. Surface modifications for antifouling membranes. Chemical

587 reviews.110:2448-2471.

588 Ridgway H, Kelly A, Justice C, Olson B. 1983. Microbial fouling of reverse-osmosis

589 membranes used in advanced wastewater treatment technology: chemical,

590 bacteriological, and ultrastructural analyses. Applied and Environmental

591 Microbiology.45:1066-1084.

592 Semiao AJC, Habimana O, Cao H, Heffernan R, Safari A, Casey E. 2013. The

593 importance of laboratory water quality for studying initial bacterial adhesion during NF

594 filtration processes. Water Research.47:2909-2920.

595 Song KC, Lee SM, Park TS, Lee BS. 2009. Preparation of colloidal silver nanoparticles

596 by chemical reduction method. Korean J Chem Eng. Jan;26:153-155. 
597 Stobie N, Duffy B, McCormack DE, Colreavy J, Hidalgo M, McHale P, Hinder SJ.

598 2008. Prevention of Staphylococcus epidermidis biofilm formation using a low-

599 temperature processed silver-doped phenyltriethoxysilane sol-gel coating. Biomaterials.

600 Mar;29:963-969.

601 Suresh AK, Pelletier DA, Doktycz MJ. 2013. Relating nanomaterial properties and 602 microbial toxicity. Nanoscale.5:463-474.

603 Swathy JR, Sankar MU, Chaudhary A, Aigal S, Anshup, Pradeep T. 2014.

604 Antimicrobial silver: An unprecedented anion effect. Scientific Reports. Nov 24;4.

605 Tiraferri A, Elimelech M. 2012. Direct quantification of negatively charged functional 606 groups on membrane surfaces. Journal of Membrane Science.389:499-508.

607 Vanloosdrecht MCM, Lyklema J, Norde W, Schraa G, Zehnder AJB. 1987.

608 Electrophoretic Mobility and Hydrophobicity as a Measure to Predict the Initial Steps of

609 Bacterial Adhesion. Applied and Environmental Microbiology. Aug;53:1898-1901.

610 Vrouwenvelder J, Van der Kooij D. 2001. Diagnosis, prediction and prevention of

611 biofouling of NF and RO membranes. Desalination.139:65-71.

612 Vrouwenvelder J, Van Paassen J, Wessels L, Van Dam A, Bakker S. 2006. The

613 membrane fouling simulator: a practical tool for fouling prediction and control. Journal

614 of Membrane Science.281:316-324.

615 Yin J, Yang Y, Hu ZQ, Deng BL. 2013. Attachment of silver nanoparticles (AgNPs)

616 onto thin-film composite (TFC) membranes through covalent bonding to reduce

617 membrane biofouling. Journal of Membrane Science. Aug 15;441:73-82.

618 Zhu WY, Li Z, Zhou Y, Yan XL. 2016. Deposition of silver nanoparticles onto two

619 dimensional $\mathrm{BiOCl}$ nanodiscs for enhanced visible light photocatalytic and biocidal

620 activities. Rsc Adv.6:64911-64920.

621 Zodrow K, Brunet L, Mahendra S, Li D, Zhang A, Li Q, Alvarez PJ. 2009. Polysulfone

622 ultrafiltration membranes impregnated with silver nanoparticles show improved

623 biofouling resistance and virus removal. Water Research.43:715-723.

624 
625 Captions to Tables and Figures.

626 Table 1. The comparison of mean flux and salt rejection of pristine and modified NF270

627 membranes at 3 bar and cross-flow velocity of $0.66 \mathrm{~L} \mathrm{~min}^{-1}$ at $20{ }^{\circ} \mathrm{C}$.

628 Table 2. Surface coverage (\%) of adhered $P$. fluorescens cells on pristine and silver-

629 modified NF270 membranes following $30 \mathrm{~min}$ adhesion under nanofiltration conditions

630 (3 bar; $0.66 \mathrm{~L} \mathrm{~min}^{-1}$; Re: $579 ; 20^{\circ} \mathrm{C}$ ). Errors represent standard error of mean.

631

632 Figure 1. High resolution XPS data of the XPS survey spectra were collected in the

633 binding energy range of 0-1200 eV of pristine and silver-modified NF270 membranes.

634 The peak for silver (Ag) is depicted by a red arrow at $368 \mathrm{eV}$.

635

636 Figure 2. Adhesion profiles of Pseudomonas fluorescens and Staphylococcus

637 epidermidis cells on either pristine or silver-modified following static following $30 \mathrm{~min}$

638 on static bioadhesion assays. Presented results characterise the adhesion profile as

639 surface coverage onto membranes (A) or the antimicrobial effect of silver modified

640 NF270 as depicted by calculated dead to live ratios (B). Error bars represent standard

641 error of the mean.

642

643 Figure 3. Scanning electron micrographs of fouled NF270 membranes following

644 nanofiltration at 3 bar and cross-flow velocity of $0.66 \mathrm{~L} \mathrm{~min}^{-1}$ at $20{ }^{\circ} \mathrm{C}$. Representative

645 micrographs were obtained depicting the structural integrity of $P$. fluorescens cells on

646 either pristine (A-B) or silver-modified (C-D) membranes following $30 \mathrm{~min}$

647 nanofiltration processes.

648

649 Figure 4. The effect of saline environments on leaching of silver-modified NF270. The

650 cumulative release of silver from silver modified NF270 membrane was assessed

651 following exposure to Milli-Q water or Raw water medium under non-permeate

652 conditions at a cross velocity of $0.037 \mathrm{~m} \mathrm{~s}^{-1}, \mathrm{Re}=14.32$ and shear rate of $536 \mathrm{~s} \mathrm{~s}^{-1}$. 
654 Figure 5. The effect of nanofiltration processes on the concentration of silver-modified 655 NF270 membranes. Silver concentrations $\left(\mathrm{mg} \mathrm{cm}^{-2}\right)$ of surface modified membranes $i$ ) 656 prior to, ii) following 2-hour compaction at 15 bar with Milli-Q and iii) 30 min salt 657 conditioning at 3 bar with Raw water, are presented following standard nanofiltration 658 experimental conditions.

659 